\title{
General Psychiatry CUMS and dexamethasone induce depression-like phenotypes in mice by differentially altering gut microbiota and triggering macroglia activation
}

Jing Wu (1) , ${ }^{1}$ Jinhang Li, ${ }^{1}$ Chhetri Gaurav, ${ }^{1}$ Usman Muhammad, ${ }^{1}$ Yantian Chen, ${ }^{1}$ Xueyi Li, ${ }^{1}$ Jinghong Chen (1) ${ }^{2,3}$ Zejian Wang ${ }^{1}$
To cite: Wu J, Li J,

Gaurav C, et al. CUMS and dexamethasone induce depression-like phenotypes in mice by differentially altering gut microbiota and triggering macroglia activation. General Psychiatry 2021;34:e100529. doi:10.1136/ gpsych-2021-100529

- Additional supplemental material is published online only. To view, please visit the journal online (http://dx.doi.org/10.1136/ gpsych-2021-100529).

Received 05 May 2021 Accepted 19 0ctober 2021

Check for updates

(C) Author(s) (or their employer(s)) 2021. Re-use permitted under CC BY-NC. No commercial re-use. See rights and permissions. Published by BMJ.

${ }^{1}$ School of Pharmacy, Shanghai Jiao Tong University, Shanghai, China

${ }^{2}$ Shanghai Mental Health Center, ShangHai Jiao Tong University School of Medicine, Shanghai, China

${ }^{3}$ Shanghai Key Laboratory of Psychotic Disorders, ShangHai Jiao Tong University School of Medicine, Shanghai, China

Correspondence to Dr Zejian Wang; wangzejian@sjtu.edu.cn

Dr Jinghong Chen; chenjh_008@hotmail.com

\section{ABSTRACT}

Background Although the link between gut microbiota and depression has been suggested, changes of gut microbiota vary largely among individuals with depression.

Aims Explore the heterogeneity of microbiota-gut-brain axis and new pathogenic characteristics in murine models of depression.

Methods Adolescent female mice were randomly divided into control (CON) group $(n=10)$, chronic unexpected mild stress (CUMS) group $(n=15)$ and dexamethasone (DEX) group $(n=15)$. Mice in the DEX group were gavaged twice a day with $0.2 \mathrm{mg} / \mathrm{kg}$ of DEX for 5 weeks, whereas CON mice were given the same amount of solvent. Mice in the CUMS group were exposed to stressors. After behavioural evaluations, all mice were sacrificed for harvesting tissues and blood samples. Enzyme-linked immunosorbent assay (ELISA) was conducted for measuring levels of corticosterone (CORT) and interleukin-1 $\beta$ (IL-1 $\beta)$ in sera, whereas levels of protein expression in colon and hippocampal tissues were examined by western blot. Faecal microbial communities were analysed by sequencing $16 \mathrm{~S}$ rDNAs.

Results Mice in CUMS and DEX groups exhibited severe depression-like behaviours. Compared with CON mice, CUMS-exposed mice showed a significant increase in both $\alpha$ and $\beta$ diversity. Prevotellaceae and Desulfovibrio were enriched, whereas Bacilli were decreased in the faeces of mice in the CUMS group. DEX-treated mice had a decrease in the abundance of Clostridium XVIII. Levels of occludin in colon tissue of DEX-treated mice were reduced. Relative to mice in the CON and CUMS groups, DEX-treated mice contained higher serum levels of CORT and IL-1 $\beta$. Compared with CON mice, mice in the DEX and CUMS groups had higher levels of IL-1 $\beta$ in sera and lower levels of glial fibrillary acidic protein (GFAP), Nestin, Synapsin-1 and $\mathrm{P} 2 \mathrm{Y} 12$ receptor in the hippocampus.

Conclusions Changes of gut microbiota diversity, intestinal integrity and neuroinflammation in the brain contribute to CUMS-induced depression, whereas pathobionts and excessive immunosuppression with damaged neuronal synapses is a basis of the DEX-induced depression.

\section{INTRODUCTION}

Depression is characterised by depressive mood and loss of interest in daily activities. ${ }^{1}$ According to the World Health Organization (WHO), there are more than 350 million people of all ages suffering from depression, making it a leading cause of disability over any other diseases. ${ }^{2}$ Up to now, pharmacological, psychological and physical interventions are the mainstay of depression treatment. ${ }^{3}$ However, a significant population of persons with depression do not respond to these treatments. Depression in children and younger individuals can devastatingly impact their development, ability to adapt and performance at school. ${ }^{5}$ It is documented that most antidepressants are unable to produce a satisfactory result for young patients. Therefore, further elucidation of the mechanisms that underlie depression is needed.

The microbiota-gut-brain axis, through which the gut and the brain communicate to each other, has been regarded as a great example of biopsychosocial interplay in the pathogenesis of neurological diseases. ${ }^{6} \mathrm{Accu}-$ mulating evidence suggests that changes in the metabolic states of the host influence the composition of gut microbiota. While varied composition of gut microbiota in patients with depression or animal models of depression has been widely reported, there exists inconsistencies regarding the characteristics of gut microbiota in people with depression compared with healthy adults. Even conflicting results are reported on microbial diversity as well as the abundance of bacteria at phyla, family and genus taxonomic levels in people with depression. Hence, it is necessary to further investigate the role of microbiota-gut-brain axis in the development of depression. 
Women are more susceptible to developing depression and anxiety disorders in response to stress than men. Dysfunction of the hypothalamic-pituitary-adrenal (HPA) axis plays a central role in the formation of depression in women. ${ }^{7}$ There is evidence that depression is more prevalent among females with abdominal obesity and overactivation of the HPA axis. ${ }^{8}$ Additionally, approximately $50 \%$ to $75 \%$ of individuals with excessive weight loss due to anorexia nervosa experience lifetime major depressive disorder (MDD) ${ }^{9}$ We speculate that these subtypes of major depression may arise from alterations in the microbiota-gut-brain axis. In this study, we used adolescent female mice to model depression with two different inducers, specifically dexamethasone (DEX) and chronic unexpected mild stress (CUMS), and then compared the heterogeneity and homogeneity of gut microbiota. We found that there were significant changes in gut microbiota, expression levels of tight junction proteins in the colonic mucosa, as well as signs of neuroinflammation in the hippocampus in mice with depressive behaviours induced by CUMS or DEX. Our study suggests that analysis of the heterogeneity of gut microbiota is helpful to our understanding of depression pathogenesis and to the discovery of targets for treating depression in women.

\section{MATERIALS AND METHODS \\ Reagents}

Antibodies for glial fibrillary acidic protein (GFAP), Nestin, and Caspase-1 were from Proteintech (Chicago, Illinois, USA). Antibodies against interleukin-1 $\beta$ (IL-1 $\beta$ ), glyceraldehyde-3-phosphate dehydrogenase (GAPDH) and horseradish peroxidase conjugated antirabbit and mouse IgG were acquired from Cell Signaling Technology (Boston, Massachusetts, USA). Anti-Claudin-1 and anti-Synapsin-1 were purchased from Bioworld (Dublin, Ohio, USA), whereas anti-Occludin was from
Thermofisher (USA). Anti-NLRP3 (nucleotide-binding domain-like receptor protein3) and anti-P2Y12 receptors were purchased from NOVUS (Cambridge, UK) and Abcam (Cambridge, UK), respectively.

\section{Animals}

All experiments were conducted in 4 to 6 -week-old female C57/BL6 mice (14-16g), which were purchased from Shanghai SLAC Laboratory Animal, Shanghai, China. Mice were maintained in a controlled environment at $22^{\circ} \mathrm{C} \pm 1^{\circ} \mathrm{C}$ with a $12 / 12$ hour light-dark cycle. Mice were randomly divided into control $(\mathrm{CON})$ group $(n=10)$, DEX group $(n=15)$ and CUMS group $(n=15)$.

\section{Establishment of depression models}

Dysfunction of the HPA axis in female mice was induced by gavage feeding $2 \mathrm{mg} / \mathrm{kg}$ DEX twice a day for 5 weeks (figure 1). The CUMS-induced model was developed as previously described. ${ }^{10}$ In brief, mice were individually caged and subjected to CUMS for 5 weeks with the following stressors: $4^{\circ} \mathrm{C}$ ice-water swimming for $5 \mathrm{~min}$, cage tilted by $30^{\circ}$ for 24 hours, foot electric shocked for twice $(0.8 \mathrm{~mA}, 5 \mathrm{~s})$, fasting 24 hours, continuous illumination, behavioural restrictions for 2 hours, wet pad for 24 hours, tail-clamp for $90 \mathrm{~s}$, water deprivation for 24 hours and cage shaking for $15 \mathrm{~min}$. To prevent habituation and to provide an unpredictable feature to the stressors, stressors were randomly administered every day for 35 days without the same kind of stimulus. Control mice were fed normally and fed by gavage with the same volume of water.

\section{Behavioural testing}

Behaviours of all mice in each group were evaluated once CUMS and/or DEX exposure was completed. Mice were allowed to adapt to their environments for 1 week before being evaluated. All testing apparatuses were cleaned with $70 \%$ alcohol and distilled water to eliminate residual

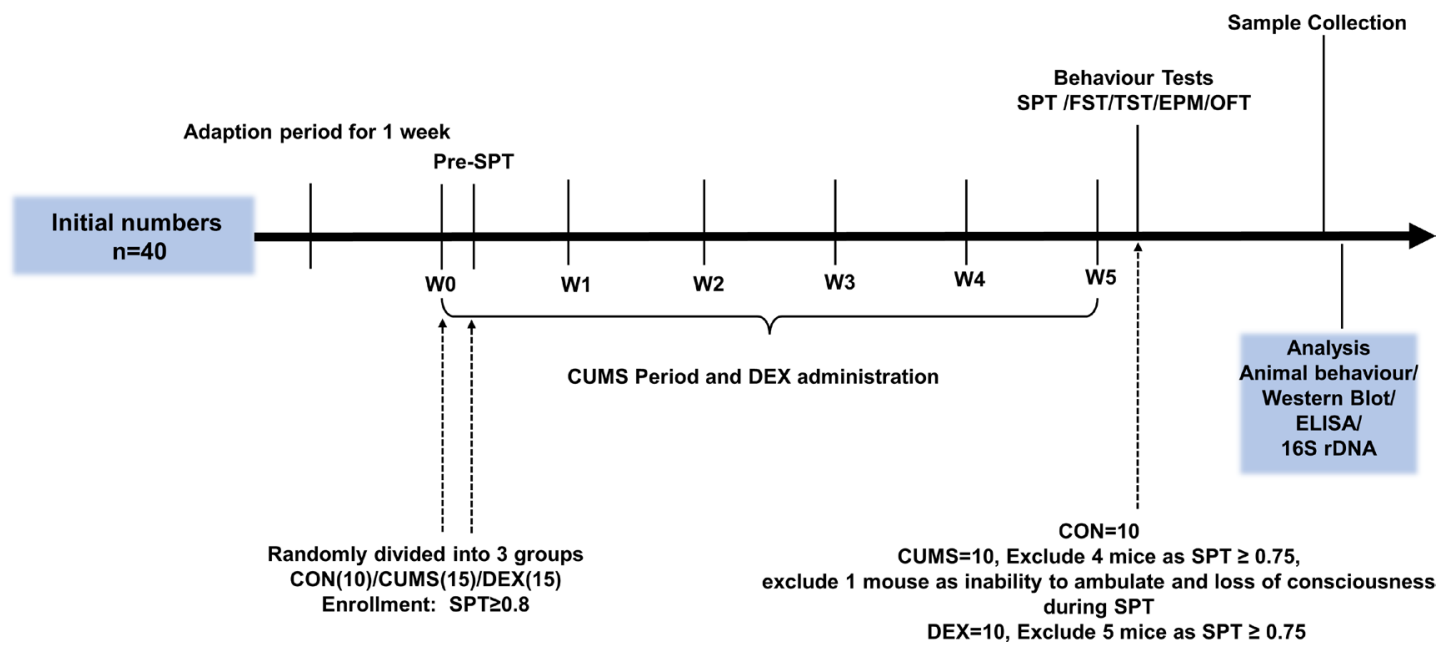

Figure 1 Experimental schedules. After environment adaption for 1 week, animals were randomly divided into three groups on the last day of the week of environmental adaption (W0) and subjected to CUMS and DEX treatment for 5 weeks (W0-W5). Body weight was measured at week 1. CON, control group; CUMS, chronic unexpected mild stress; DEX, dexamethasone; ELISA, enzyme-linked immunosorbent assay; EPM, elevated plus maze; FST, forced swimming test; OFT, open field test; SPT, sucrose preference test; TST, tail suspension test. 
odours before and after each animal was assessed. The experimenters who analysed behaviour data were blinded to the treatment conditions.

Sucrose preference test (SPT) was conducted after 1-week adaption. The SPT, open field test (OFT), elevated plus maze (EPM), tail suspension test (TST) and forced swimming test (FST) were conducted after CUMS and DEX administration were completed. The schedule is shown in figure 1.

\section{Sucrose preference test}

Mice were allowed to choose from two bottles of liquids over 24 hours; one bottle contained 1\% sucrose solution, whereas the other bottle contained the same volume of pure water. The bottle positions of the two bottles were switched once 12 hours after SPT was started. The sucrose preference was determined as the percentage of the total amount of liquid consumed.

\section{Open field test}

The open field was a box $(100 \mathrm{~cm} \times 100 \mathrm{~cm} \times 30 \mathrm{~cm})$ with its floor divided into 16 squares; the central four squares were defined as the centre, whereas the rest 12 squares were considered as periphery. Each mouse in each group was placed in the centre of the open arena and allowed to explore for $5 \mathrm{~min}$. Movements of each mouse were recorded with a video-tracking system (Shanghai Mobile Data Information Technology). The total distance moved in the arena, travel speed and rearing frequency were measured.

\section{Elevated plus maze}

The apparatus $(50 \mathrm{~cm}$ height from floor) consisted of two open arms $(30 \mathrm{~cm} \times 5 \mathrm{~cm})$ and two enclosed arms $(30$ $\mathrm{cm} \times 5 \mathrm{~cm} \times 25 \mathrm{~cm})$ and was used for measuring anxietylike behaviours of animals. Each mouse was placed in the central section facing an open arm and was allowed to explore the arms for $5 \mathrm{~min}$. Activities of each mouse were recorded by video-tracking system (Shanghai Mobile Data Information Technology). The time spent in open arms was calculated.

\section{Tail suspension test}

Each mouse was suspended upside down by fixing the tip of its tail with adhesive tape on a metal $\operatorname{rod} 40 \mathrm{~cm}$ above the floor. During the 6 min test period of testing, activities of the mice tested were recorded with a video camera. The immobility time within the last $4 \mathrm{~min}$ was calculated.

\section{Forced swimming test}

Each mouse in each group was placed in a vertical transparent cylinder $(30 \mathrm{~cm}$ in height and $12 \mathrm{~cm}$ in diameter) containing tap water $\left(25^{\circ} \mathrm{C} \pm 1^{\circ} \mathrm{C}\right.$ and $20 \mathrm{~cm}$ in depth). Activities of the mice tested over $6 \mathrm{~min}$ period of testing were recorded with a video camera. The immobility time within the last $4 \mathrm{~min}$ was calculated.

\section{Specimens collection}

After all behaviour tests were completed, mice were sacrificed by exsanguination. Before being sacrificed, faecal samples were collected in sterilised tubes. Blood samples were collected by puncturing the abdominal aorta. Sera from each mouse were extracted with a centrifugation of $5000 \mathrm{~g}$ for $15 \mathrm{~min}$ and stored at $-80^{\circ} \mathrm{C}$. Colon and hippocampal tissues were dissected on ice immediately after euthanisation. Colon tissues were cut open longitudinally and washed with ice-cold phosphate buffer saline (PBS) to remove gut contents. All tissues were all frozen and stored at $-80^{\circ} \mathrm{C}$ until being used for studies.

\section{ELISA}

Serum levels of corticosterone (CORT) and IL-1 $\beta$ were measured using mouse CORT and IL-1 $\beta$ ELISA KIT (XLPCC company), respectively, according to the supplier's instructions. In brief, after reagents were restored to room temperature, $50 \mu \mathrm{L}$ of each sample or standard as well as reagents were added into wells of a microplate sequentially and gently mixed. After incubation for 1 hour in $37^{\circ} \mathrm{C}$ incubators, samples in each well were washed five times using the washing buffer. To each sample (well), $50 \mu \mathrm{L}$ reagent $\mathrm{A}$ and $\mathrm{B}$ were added and incubated until the reaction was stopped by adding stop buffer. The absorbance at $450 \mathrm{~nm}$ was measured in a plate reader (Biotek, USA).

\section{Western blot analysis}

Tissues were lysed with ice-cold radioimmunoprecipitation assay (RIPA) lysis buffers (Beyotime Institute of Biotechnology, Jiangsu) supplemented with protease and phosphatase inhibitors (Roche, Indianapolis, Indiana, USA). Lysates were centrifuged at $4^{\circ} \mathrm{C} 12000 \mathrm{~g}$ for $10 \mathrm{~min}$. The resulting supernatants were collected. Protein concentrations were determined using Bradford reagents (Beyotime Institute of Biotechnology). An aliquot of proteins was mixed with sodium dodecyl sulfate-polyacrylamide gel electrophoresis (SDS-PAGE) sample buffers, incubated at $95^{\circ} \mathrm{C}$ for $8 \mathrm{~min}$ and separated on $10 \%$ SDS-polyacrylamide gels and transferred onto polyvinylidene difluoride membranes (Millipore, Bedford, Massachusetts, USA). The membranes blots were blocked with 5\% bovine serum albumin (BSA) in Tris-buffered saline containing 1\% Tween-20 (Tris-buffered salin with Tween-20, TBST) for 2 hours at room temperature and then incubated with primary antibodies overnight at $4^{\circ} \mathrm{C}$. Primary antibodies used for western blot analysis included: anti-claudin-1, anti-Occludin, anti-IL-1 $\beta$, anti-NLRP3, anti-Caspase-1, anti-Nestin, anti-GFAP, anti-synapsin-1 and anti-GAPDH. After washing in TBST, the blots were incubated with corresponding horseradish peroxidase (HRP)-linked secondary antibodies. Protein bands on the blots were detected with chemiluminescence detection reagents (Beyotime Institute of Biotechnology). Optical densities of interested protein bands were obtained using a software (Bio-Rad Laboratory, USA) and protein levels were normalised to GAPDH. 


\section{Fecal microbiota analysis}

The 16S rDNA identification technology was used for detecting and identifying the composition of intestinal flora of mice through services at the Shanghai Ruiyi Biological Technology Company. The quantity and quality of DNA samples isolated from faeces were examined with Thermo Nanodrop 2000 UV microspectrophotometer and gel electrophoresis, respectively. The V3-V4 region of 16S rDNA was chosen for amplification using the universal primers 341F: 5' CCTACGGGRSGCAGCAG 3' and 806R: 5' GGACTACVVGGGTATCTAATC 3'. The index sequence and linker sequence for Illumina Miseq PE250 sequencing were added to the $5^{\prime}$ end of the universal primer. PCR was performed using the highfidelity enzyme of Kapa Hifi Hotstart ReadyMix PCR Kit. PCR products were detected by agarose gel electrophoresis and recovered using the gel cutting with AxyPrep DNA Gel Recovery Kit. The quality of the library was determined by using a Thermo Nanodrop 2000 UV spectrophotometer and gel electrophoresis. Qubit was used for library quantitation. According to the data volume requirements of each sample, the corresponding proportion was mixed, and Illumina Miseq PE250 was used for computer sequencing.

\section{Alpha diversity analysis}

Alpha diversity was calculated using QIIME (V.1.9.1) software, and the corresponding dilution curve was drawn. The dilution curve uses the relative ratios of various operational taxonomic units (OTUs) known in the measured $16 \mathrm{~S}$ rDNA sequence to calculate the expected values of each Alpha diversity index when $n$ Reads are extracted ( $\mathrm{n}$ is less than the total number of measured Reads sequences), and then the curve is made according to the expected values of a set of $n$ values (generally a set of isograms less than the total number of sequences) and the corresponding alpha diversity index. Rank sum test analysis was carried out on each index of alpha diversity (Wilcox. test function in R.V3.5.1 was used for comparison between two groups of samples, and Kruskal. test function in RV.3.5.1 was used for comparison between two groups of samples previously), and Alpha diversity index with significant difference under different conditions was screened by rank sum test.

\section{Beta diversity analysis}

\section{Principal component analysis}

In order to further show the difference of species diversity among samples, principal coordinate analysis (PCoA) was used to show the difference between samples implemented with the Dudi.pco function in the R (V.3.5.1) language ade 4 package.

\section{Anosim analysis}

Anosim (similarity) analysis is a non-parametric test to test whether the difference between groups (two or more groups) is significantly greater than the difference within the group, so as to determine whether the grouping is meaningful. First, the distance between two samples was calculated by Unifrac algorithm, and then all distances were sorted from small to large. The calculation process is realised by anosim function in the vegan package of $\mathrm{R}$ (V.3.5.1) language.

\section{LEfSe analysis}

Linear discriminant analysis (LDA) effect size (LEfSe analysis V.1.0): LEfSe uses LDA to estimate the impact of each component (species) abundance on the difference effect and to identify communities or species that have significant differences in sample partitioning. The analysis was performed using LEfSe Tools.

\section{Statistical analysis}

Data were expressed using mean (standard deviation, SD). The SPSS statistical software V.16.0 was used for statistical analysis. One-way analysis of variance followed by the Tukey's test was conducted for comparisons. To reveal differentially abundant features, relative abundance comparisons at levels of genus, family and phylum were performed on non-normalised data, employing non-parametric Mann-Whitney U tests, as well as, Kruskal-Wallis tests with multiple comparison correction. A value of $\mathrm{p}<0.05$ was considered as having statistical significance.

\section{RESULTS}

CUMS and DEX treatments induce depression-like behaviours in female mice

We evaluated behaviours of mice with SPT, OFT, EPM, TST and FST to determine whether CUMS and DEX evoked depression-like or anxious behaviours. Figure 2 showed that mice in both CUMS and DEX groups displayed a significant decrease in the total travel distance, movement speed, frequency of rearing and crossing in open arena, as well as the time spent in open arms of the EPM and sucrose preference compared with the mice in the CON group $(p<0.01)$. Mice exposed to CUMS or DEX had a significant increase of immobility time in TST and FST relative to mice in the CON group $(\mathrm{p}<0.01)$. While there was no significant difference in body weight between groups at the beginning of experiments (figure 2B), the body weight of mice $(p<0.01)$ was significantly reduced and increased, respectively, relative to that of mice in the CON group $(p<0.01)$. Collectively, these data suggest that we have succeeded in establishing CUMS and or DEX induced models of depression.

\section{Differences in the gut microbiota diversity in two mouse models of depression}

To determine whether gut microbiota was altered in CUMS and or DEX treated mice, we collected faeces for analysing bacterial diversity and composition. The dilution curve in online supplemental figure S1 
A
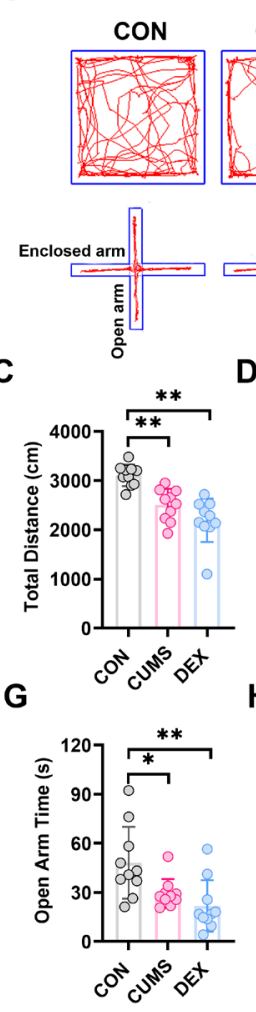
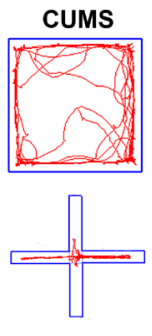

D

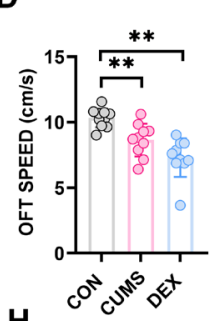

$\mathrm{H}$

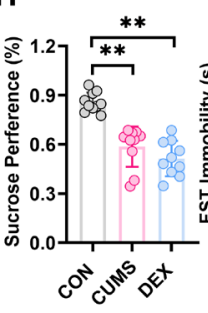

B

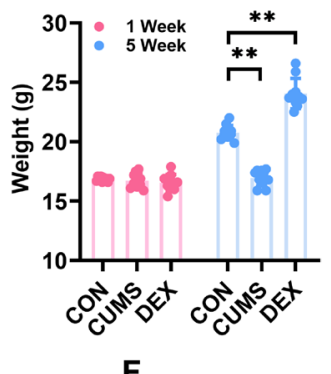

E
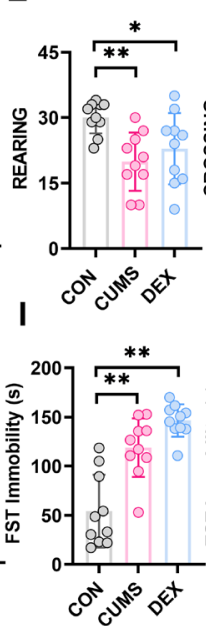

$F$
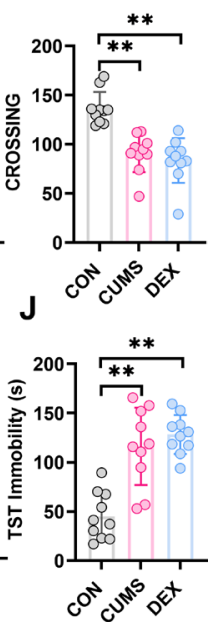

Figure 2 CUMS and DEX treatments alter body weight and behavioural performances of mice. (A)Automated tracking in the large open field and the elevated plus-maze test. (B) Body weight of each mouse in each group was measured at week 1 and the last day of CUMS or DEX treatment and graphed. The weight of mice subjected to CUMS was less than the CON group and the DEX group gained more weight than the CON group after 5 weeks. Total distance, moving speed, frequency of rearing and crossing of mice in OPT (C-F) and time spent in open arms of EPM (G) were significantly decreased. (H)The sucrose preference test decreased notably in the CUMS and DEX group. The time of FST immobility (I) and TST immobility (J) were significantly increased. Data are shown as mean(SD) ( $n \geq 3$ in each group). ${ }^{*} p<0.05$; ${ }^{\star \star} p<0.01$, versus control group. CON, control; CUMS, chronic unexpected mild stress; DEX, dexamethasone; EPM, elevated plus maze; FST, forced swimming test; OFT, open field test; TST, tail suspension test.

showed that the sequencing depth covered all species in the samples. The $\alpha$ diversity indices including chao1, observed species, shannon and simpson were used as indicators of species richness and uniformity in community ecology. As shown in online supplemental figure S2, there was a significant difference in the abundance and diversity between the CON group and CUMS group $(\mathrm{p}<0.05)$, whereas there was no significant difference between the CON group and the DEX group $(p>0.05)$. These data suggest that CUMS but not DEX alters the abundance and richness of intestinal flora. $\beta$ diversity analysis was used for comparing the differences in species diversity of a pair of intergroup samples. Anosim similarity revealed the differences between intergroups

and intragroups. The differences of intergroups were significantly greater than those of intragroups (online supplemental figure $\mathrm{S} 3, \mathrm{R}=0.892, \mathrm{p}=0.007$; $\mathrm{R}=0.524, \mathrm{p}=0.01)$. To further compare the species diversity, PCoA was conducted (online supplemental figure S4). ${ }^{112}$ As shown in figure 3 , the relative abundance in the CUMS group was higher than that in the CON group, but the relative abundance of the DEX group was similar to that in the CON group. These results indicate that gut microbiota of mice subjected to CUMS are significantly different from the CON group, whereas the compositions of intestinal flora between DEX and CON groups are similar.

\section{The species abundance and difference of gut microbiota in CUMS and DEX group}

According to the results of species annotation, the corresponding histogram of each sample was analysed at phylum, class, order, family and genus classification levels respectively, so as to intuitively discern the abundance and proportion of species in different classification levels of each sample. At the phylum level, the abundance of Bacteroidetes (72.86 (2.43)\%) and of Firmicutes (24.81 (4.24)\%) in the CUMS group was higher and lower, respectively, when compared with the CON group (43.25 (14.17)\%). There was no significant difference in the abundances of Bacteroidetes and Firmicutes between the DEX and CON groups. At the genus level, the abundances of Bacteroides $(p<0.01)$ and Parabacteroides $(p<0.01)$ were decreased significantly in the DEX group relative to that in the CON group. The abundance of Parabacteroides $(\mathrm{p}<0.01)$ as well as Lactobacillus and Staphylococcus $(\mathrm{p}<0.05)$ was decreased in the CUMS group. Escherichia/Shigella was found in the DEX group but not the CUMS or the CON group. Moreover, the relative abundances of Alloprevotella, Desulfovibrio, Parasutterella and Flavonifractor were significantly increased in the CUMS group relative to their corresponding abundances in the CON group. LDA effect size (LEfSe) was used to evaluate the impact of the abundance of each component and to identify the communities or species that are different between groups. Compared with the control group, mice in the CUMS group had an increase of the genera in their intestinal microflora, which included:Prevotellaceae, Alloprevotella, Oscillibacter, Desulfovibrionaceae, Mucispirillum, Olsenella, Allobaculum, Candidatus Saccharibacteria and Saccharibacteria genera incertae sedis, and a decrease of Bacilli, Lachnospiracea incertae sedis, Neisseriaceae, Neisseriales, Neisseria, Eubacteriaceae, Eubacterium, Butyricicoccus, Staphylococcus, Bacillales and Staphylococcaceae, Compared with the control group, mice in the DEX group had an increase of Enterobacteriaceae, Escherichia Shigella, Gammaproteobacteria, Enterobacteriales, Anaerofustis and Erysipelotrichaceae_incertae sedis and a decrease of Clostridium XVIII. Relative to those in the CUMS group, Prevotellaceae, Alloprevotella, Ruminococcaceae, Oscillibacter, Desulfovibrionaceae, Deltaproteobacteria, Desulfovibrionales, Desulfovibrio, Deferribacteres, Deferribacteraceae, Deferribacterales, Burkholderiales, Mucispirillum, Olsenella, Allobaculum, Sutterellaceae, Candidatus Saccharibacteria and Saccharibacteria genera incertae sedis were significantly decreased, whereas Eubacteriaceae, Eubacterium, Enterobacteriaceae, Escherichia/ 
A
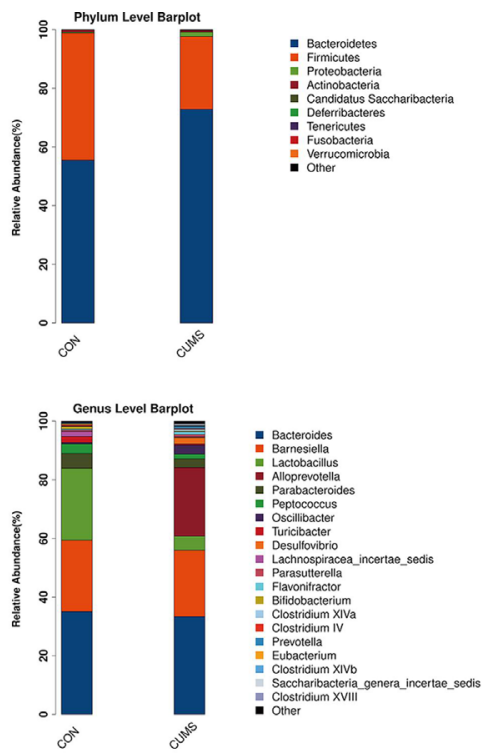
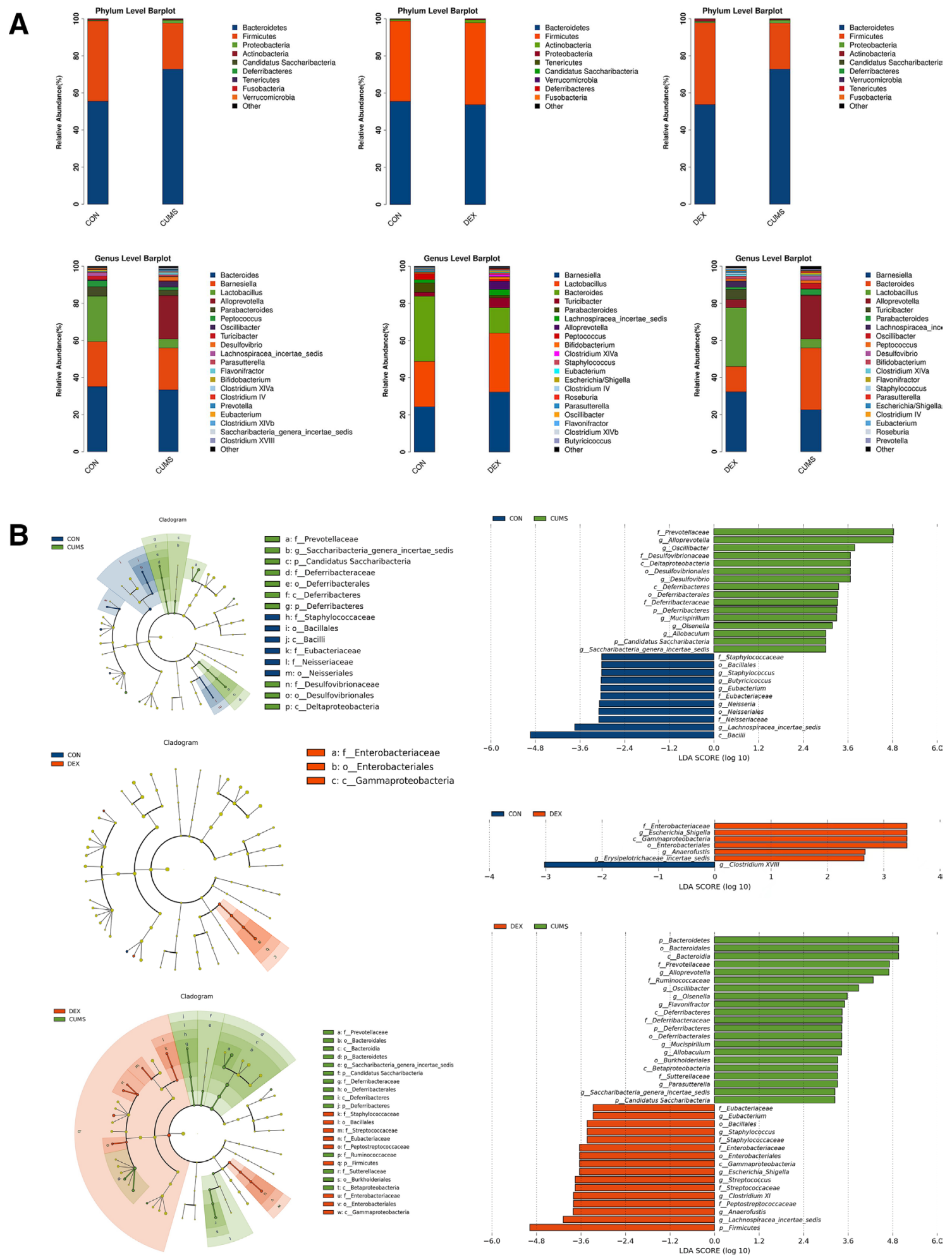

Figure 3 Differential composition of gut microbiota in two depression models. (A) Comparison of the abundance of microbial phylum and genus in CON, CUMS and DEX groups . LDA score plot and cladogram plot from LEfSE analysis of the gut microbiota composition in CON, CUMS and DEX groups at baseline and end point. (B) Microbial taxa shown have an LDA score higher than 2 . CON, control; CUMS, chronic unexpected mild stress; DEX, dexamethasone; LDA, linear discriminant analysis; LEfSE, linear discriminant analysis effect size.

Shigella, Gammaproteobacteria, Streptococcus, Enterobacteriales, Anaerofustis, Erysipelotrichaceae_incertae_sedis, Streptococcaceae, Peptostreptococcaceae, Lachnospiracea_incertae_sedis, Clostridium $X I$ and Firmicutes were increased in the DEX group.
The intestinal barrier integrity and proinflammatory cytokine in the colon of depression mouse models

The intestinal epithelial barrier is the first line of defence of the host. Serious inflammation or many other intestinal 
A

Colon

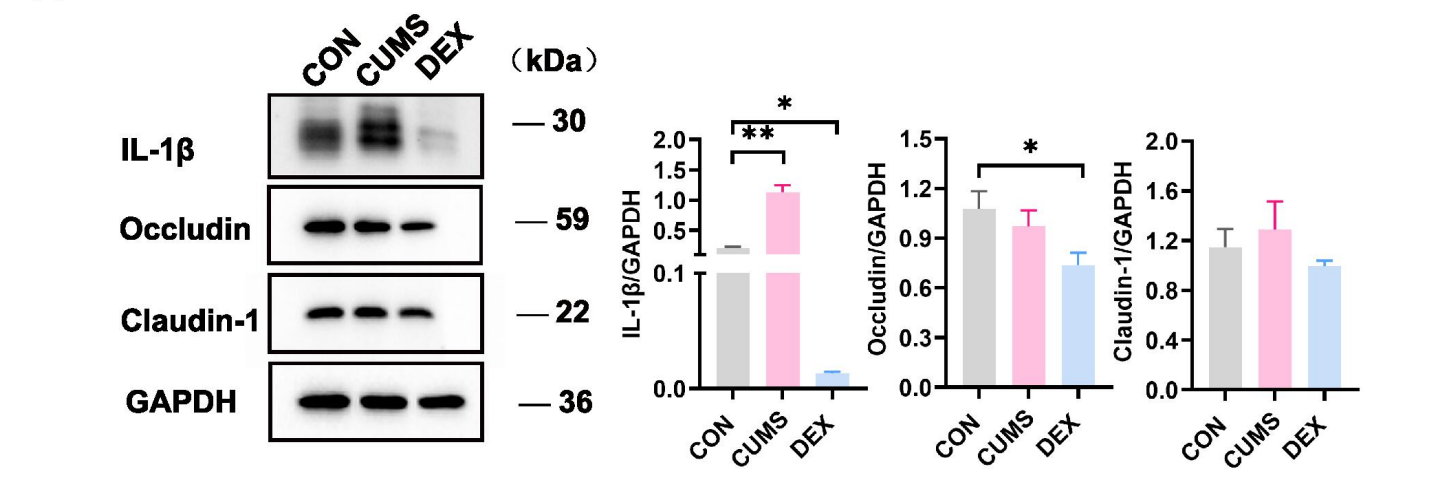

B

Serum

CORT

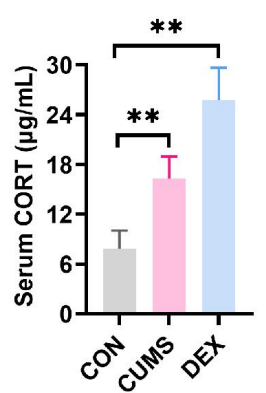

IL-1 $\beta$

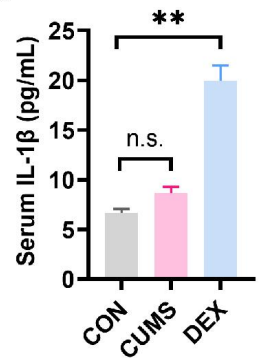

Figure 4 Expression levels of proteins related to inflammation were changed in colon tissues and peripheral blood. (A) Western blot analyses of lysates prepared from colon tissues of mice in each group with indicated antibodies. The blots shown are representative of at least three independent studies. Bar graphs were quantitative data of western blot analyses and showed altered expressions of occludin, claudin-1 and IL-1 $\beta$ in the colon of mice treated with CUMS or with DEX. (B) Levels of CORT and IL-1 $\beta$ in peripheral bloods of mice in each group were measured and graphed. Data were shown as mean(SD). In both figure parts $A$ and $B,{ }^{*} p<0.05$; ${ }^{* *} p<0.01$; n.s., no significance versus control group. CON, control; CORT, corticosterone; CUMS, chronic unexpected mild stress; DEX, dexamethasone; GAPDH, glyceraldehyde-3-phosphate dehydrogenase; IL-1ß, interleukin-1 $\beta$.

diseases are associated with the integrity damage of the intestinal epithelial barrier. Accumulating evidence suggests that patients with chronic colon inflammation frequently have serious depression and anxiety morbidities. ${ }^{13}$ Therefore, we evaluated the colon epithelial barrier integrity by examining expression levels of claudin-1 and occludin, which are protein markers of the tight junction. While levels of claudin-1 were comparable in colon tissues of mice between CON and DEX groups, levels of occludin were significantly decreased in colon tissues of mice in the DEX group relative to those in colon lysates of mice in the CON group $(p<0.05)$. Levels of IL-1 $\beta$, which reflects inflammation, were significantly increased in colon tissues of mice in the CUMS group (figure $4 \mathrm{~A}, \mathrm{p}<0.01$ ). These results suggest that DEX influences the colon permeability, whereas CUMS evokes inflammation in the gut.

The heterogeneity of serum levels of CORT and IL-1 $\beta$ in CUMS and DEX groups

Glucocorticoids (GCs) are stress hormones released by the adrenal cortex and regulate body metabolism and function. The serum level of corticosterone (CORT) is considered to be an indicator of stress and the severity of depression. The proinflammatory cytokine IL-1 $\beta$ is a cytokine released by macrophages on lipopolysaccharide (LPS)-mediated stimulation of toll-like receptor 4 (TLR4). Compared with that in the control group, the serum level of CORT was significantly increased in the CUMS group $(\mathrm{p}<0.01)$. Noticeably, serum levels of both CORT and IL-1 $\beta$ were significantly elevated in the DEX group relative to their levels in CON and CUMS groups (p-values <0.01). The rise of CORT in the serum of mice in the DEX group may partly result from metabolites of dexamethasone (figure 4B).

\section{CUMS and DEX groups mainly affected glia cells instead of} neuron in the hippocampus

The hippocampus has been regarded as the main target in the formation of depression. ${ }^{14}$ Compared with those of the CON group, levels of GFAP and Nestin in the hippocampus of mice in both CUMS and DEX groups were significantly decreased ( $p$-values $<0.05$ ). No significant difference in levels of neuron-specific neuronal nuclei $(\mathrm{NeuN})$ among the three groups was noted $(\mathrm{p}>0.05)$. However, we found that levels of synapsin-1 and P2Y12 were decreased in the hippocampi of mice in both CUMS and DEX groups when compared with those in the CON 

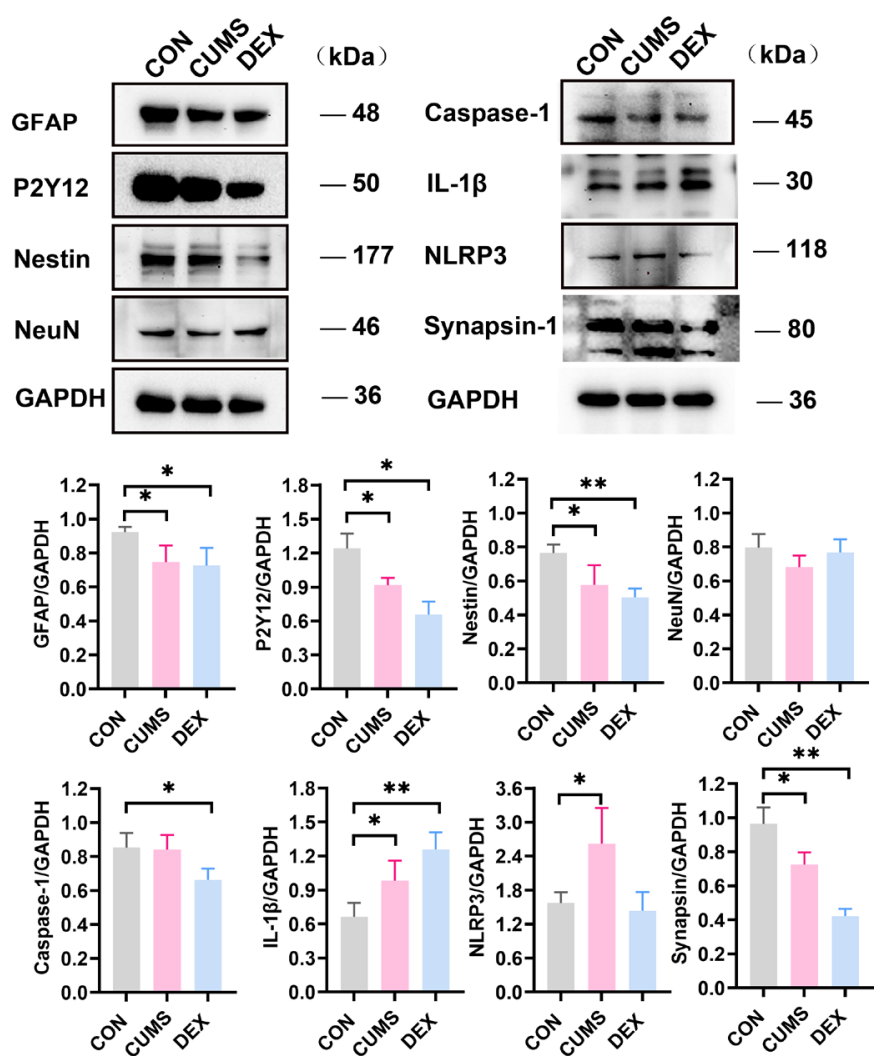

Figure 5 Differential protein expressions of GFAP, P2Y12, Nestin, NeuN, Caspase-1, IL-1ß, NLRP3 and Synapsin-1 in the hippocampus of two depression models. (A) Lysates were prepared from hippocampal tissues of mice in each group and analysed by SDS-PAGE and western blot with indicated antibodies. (B-I) Shown are data from one animal in each group. Data are shown as mean (SD) $(n=3$ in each group). ${ }^{*} p<0.05$; ${ }^{\star *} p<0.01$, versus control group. CON, control; CUMS, chronic unexpected mild stress; DEX, dexamethasone; GFAP, glial fibrillary acidic protein; IL-1 $\beta$, interleukin-1 $\beta$; NeuN, neuronal nuclei; NLRP3, nucleotidebinding domain-like receptor protein3; SDS-PAGE, sodium dodecyl sulfate-polyacrylamide gel electrophoresis.

group (figure 5, $\mathrm{p}<0.05$ ). Compared with their expression in the control group, NLRP3 and IL-1 $\beta$ were upregulated in the CUMS group, but only IL-1 $\beta$ was found to increase in the hippocampus of mice in the DEX group $(\mathrm{p}<0.05)$. Collectively, these results suggest that the activation of glial cells occurs in the brain of CUMS or DEX induced mouse model of depression.

\section{DISCUSSION}

\section{Main findings}

The microbiota-gut-brain axis is emerging as a particular area of interest and a potential new target for the treatment of central nervous system disorders. Dynamic homeostasis of the intestinal environment is maintained by the inter-relation and interaction between beneficial and pathogenic microbes in the intestinal tract. The dysbiosis could cause long-lasting harmful effects to health by releasing enteric toxins, invading the gut mucosa, triggering inflammation and even interfering with nutrient absorption. Considerable studies have shown that chronic low-grade inflammation increases the susceptibility to depression. We found that compared with the CON group, the CUMS group had a significant change in both $\alpha$ and $\beta$ diversities of intestinal microbiota, suggestive of dysbiosis in the gut. However, DEX exhibited little effect on the microbiota community. An elevation in the Firmicutesto-Bacteroidetes ratio signs a greater energy-harvesting capacity from undigested carbohydrates with more lipids production. Obesity is associated with a high proportion of Firmicutes, whereas lean favours the Bacteroidetes. Our results showed that the body weight of CUMS-treated mice significantly declined and coincidently had a significant decrease in the abundance of Firmicutes-to-Bacteroidetes on the phylum level compared with the control group.

On the genus level, we found a high abundance of Alloprevotella, Desulfovibrio, Parasutterella and Flavonifractor, and a low abundance of Parabacteroides, Lactobacillus and Staphylococcus in the CUMS group. In the DEX group, Escherichia/Shigella were highly abundant, and Bacteroides and Parabacteroides were relatively scant. Parabacteroides and Lactobacillus are probiotics and contribute to the intestinal microflora balance and nutrient digestion by regulating bile acid profiles. The abundance of Lactobacillus species in the intestinal tracts of patients with major depression are significantly decreased. $^{15} 16$ Lactobacillus competes with harmful bacteria in the intestinal mucosa space and nutrition and also promotes the conversion of glutamic acid to $\gamma$-aminobutyric acid (GABA) ${ }^{17}$ GABA is one of the most important inhibitory neurotransmitters in the central nervous system and is involved in the pathogenesis of anxiety and depression associated with functional bowel disorders. ${ }^{15}$ Increased levels of Desulfovibrio and Parasutterella have been found in models of gout and in patients with inflammatory bowel disease. ${ }^{18} 19$ Overgrowth of Desulfovibrio in the gut has been suggested to play a role in regressive autism..$^{20-22}$ Flavonifractor, one of the Bacteroidales order Bacteroidia class Bacteroidetes phylum, displays a high invasion potential in the gut of inflammatory bowel diseases in patients with major depression. ${ }^{23}$ Alloprevotella is a dominant bacterium in healthy people and produces the short-chain fatty acid. As the abundance of Alloprevotella in the gut and the fasting state are correlated with each other, we speculate that the loss of body weight in the CUMS group might be associated with a higher abundance of Alloprevotella. The most isolated Enterobacteriaceae is Escherichia/Shigella, which was significantly enriched in the DEX group.

Allobacullum and Olsenella are related with intestinal protection effect by producing butyrate. Both are found to decrease in the gut of obese mice with a high fat diet. ${ }^{24-26}$ We found that Allobacullum and Olsenella were increased in the gut of mice in the CUMS group. The Staphylococcus is included in the Staphylococcaceae 
family, Bacilli class, Bacillales order and Firmicutes phylum. It is positively associated with energy intake by using amino acids as a major energy source. The decrease of it may further reduce energy utilisation in the CUMS group. ${ }^{15}$ Additionally, the increased Erysipelotrichaceae_incertae_sedis in gut microbiota composition has also been reported in people with depression. High fat diet-induced obesity is associated with high abundance of Anaerofustis. ${ }^{27}$ The abundance of Clostridium XVIII in the gut is related to food allergy. ${ }^{28}$ We found that DEX treatment significantly decreased Clostridium XVIII and increased Anaerofustis, such changes might contribute to the altered body weight of mice in the DEX group. Compared with the DEX group, the CUMS group had higher abundances of Ruminococcaceae, Burkholderiales and Sutterellaceae. Ruminococcaceae belongs to the Clostridia class, which are anaerobic and gram-positive microbes. It has been reported that the abundance of Ruminococcaceae and the abundances of Burkholderiales and Sutterellaceae are increased and decreased, respectively, in the adrenocorticotropic hormone induced rat model of depression. ${ }^{29}$ In our CUMS-induced mouse model of depression, the abundances of Burkholderiales and Sutterellaceae were increased, likely because of the high levels of oestrogen in female mice. Compared with the CUMS group, the DEX group had higher abundances of Peptostreptococcaceae, Lachnospiraceae and Clostridium XI, which are usually associated with gut inflammation and blood lipids. ${ }^{30}$

Since most microbiota are located in the colon, we evaluated the integrity of colonic mucosa of depression models via two tight junction associated proteins. DEX significantly decreased the occludin expression in the colon, which may weaken the gut mucosal barrier and facilitate the translocation of gut microbiota. DEX was orally administered in the DEX group; it may cause severe immune inhibition in gastrointestinal tract of mice, which contributed to the low level of local IL- $1 \beta$ in the colon tissue and impaired colon mucosa regeneration in the DEX group. A rise in serum CORT level is a risk factor for major depression or anxiety disorder. ${ }^{31}$ Compared with the two other groups, the mice treated with DEX had the highest serum level of CORT and IL-1 $\beta$, which may result from DEX-induced exacerbation of pathobiont infection and bacterial endotoxins with a reduced phagocytic capacity of blood leucocytes. The level of IL- $1 \beta$ was significantly higher in the colon of the CUMS group than that of the control group, which may due to the high relative abundance of opportunistic pathogens.

Considering that high levels of GCs strongly inhibit hippocampal plasticity and functions, ${ }^{32}$ we further investigated the changes of neural biomarkers in the hippocampus in each group. There was no significant difference in levels of NeuN. We observed that synapsin-1 expression was significantly decreased in the CUMS and DEX groups. Therefore, we conclude that the synaptic plasticity of hippocampus is more prone to being damaged in depression models. The low expression of GFAP and Nestin in DEX and CUMS groups suggest the dysfunction of astrocytes and neural stem/progenitor cells (NSPCs) in the depression-like behaviour mice. ${ }^{33} 34$ Astroglia cells provide neurotrophic factors for the survival of neuron and proliferation of NSPCs during the course of neurogenesis ${ }^{35}$ and maintain the balance of neurons energy metabolism via lactate shuttle. ${ }^{36}$ Dysfunctions of astrocytes and synaptic plasticity in the hippocampus are common characteristics in the two different depression models.

Neuroinflammation contributes to the pathophysiology of MDD. ${ }^{37} \mathrm{P} 2 \mathrm{Y} 12$ purinergic receptor expression is necessary for neuron-microglia interactions; its receptors on microglia are thought to be a key player in the surveillance of the local environment and neurogenesis. ${ }^{38}$ The decrease in P2Y12 receptors may aggravate the damage of hippocampal neurogenesis in the CUMS and DEX groups compared with the control group. We also found a significant increase in NLRP3 in the CUMS group and a remarkable decrease of it in the DEX group. NLRP3 inflammasome plays a key role in shaping immune/ inflammatory responses in brain diseases. ${ }^{39}$ These results suggest that inflammasome plays an important role in the hippocampal neuroinflammation in the CUMS group. However, we found a significant decrease in levels of caspase-1 and NLRP3 in the mice from the DEX group compared with CON mice. Since DEX is one of the most commonly used anti-inflammatory agents, the microglia activation and increased hippocampal IL-1 $\beta$ level in the DEX group may have originated from the damage of the neuron-microglia crosstalk in the brain and or elevated peripheral cytokines. Our results suggest the heterogeneous constitution of neuroinflammation in the formation of depression.

\section{Limitations}

Although we revealed the differences in the microbiotagut-brain axis in two common models of depression, we only focused on the neural markers in the hippocampus. It will be interesting to know if there are changes of prefrontal cortex to understand the precise mechanism of neuroinflammation in our models. The gender differences in the microbiota-gut-brain in our models also deserve additional investigation.

\section{Implications}

Up to now, studies on depression in animal models mainly involve CUMS, which simulates the pathophysiology of MDD. Repeated administration of corticosterone has been widely used to simulate behavioural changes in patients with MDD, including anhedonia, immobility and anxiety behaviour. ${ }^{40}$ Based on our findings, chronic low-grade inflammation in the peripheral and central nervous system with neural synapses loss is a common contributor to the formation of two different depression models. The increase in gut conditional pathogens with nutrient deficiency may 
play an important role in the formation of CUMSinduced depression. Excessive immune inhibition, obesity and the increase of gut pathobionts are likely to be key triggers of DEX-induced depression.

Contributors ZW designed the entire study, monitored data collection for the whole experiment, wrote the statistical analysis plan and analysed the data, and drafted and revised the paper. He is a guarantor. JW implemented the experiments and analysed the data, and drafted and revised the paper. XL revised the paper and thoroughly provided technical supports for the experimental design. JC initiated the collaborative project, co-supervised data collection for the whole trial and revised the paper. CG, UM, YC and JL provided the support to edit the manuscript and design the trial. All authors contributed to the manuscript and read and approved the final manuscript.

Funding This project was supported by the Shanghai Key Laboratory of Psychotic Disorders (N0.13dz2260500), National Natural Science Foundation of China (No. 81871122) and Interdisciplinary Program of Shanghai Jiao Tong University (ZH2018QNA59).

Competing interests None declared.

Patient consent for publication Not applicable.

Ethics approval Ethics approval Obtained (20181112). The study was approved by the Institutional Ethics Committee of the Shanghai Jiao Tong University animal experiment Center (IORG Number: 20181112). The Institutional Ethics Committee of the Shanghai Jiao Tong University also approved the consent procedure.

Provenance and peer review Commissioned; externally peer reviewed.

Data availability statement All data relevant to the study are included in the article or uploaded as supplemental information.

Supplemental material This content has been supplied by the author(s). It has not been vetted by BMJ Publishing Group Limited (BMJ) and may not have been peer-reviewed. Any opinions or recommendations discussed are solely those of the author(s) and are not endorsed by BMJ. BMJ disclaims all liability and responsibility arising from any reliance placed on the content. Where the content includes any translated material, BMJ does not warrant the accuracy and reliability of the translations (including but not limited to local regulations, clinical guidelines, terminology, drug names and drug dosages), and is not responsible for any error and/or omissions arising from translation and adaptation or otherwise.

Open access This is an open access article distributed in accordance with the Creative Commons Attribution Non Commercial (CC BY-NC 4.0) license, which permits others to distribute, remix, adapt, build upon this work non-commercially, and license their derivative works on different terms, provided the original work is properly cited, appropriate credit is given, any changes made indicated, and the use is non-commercial. See: http://creativecommons.org/licenses/by-nc/4.0/.

ORCID IDs

Jing Wu http://orcid.org/0000-0002-1028-6060

Jinghong Chen http://orcid.org/0000-0002-7428-9359

\section{REFERENCES}

1 Cui R. Editorial: a systematic review of depression. Curr Neuropharmacol 2015;13:480.

2 Smith K. Mental health: a world of depression. Nature 2014;515:181.

3 Gelenberg AJ. A review of the current guidelines for depression treatment. J Clin Psychiatry 2010;71:e15.

4 Kvam S, Kleppe CL, Nordhus IH, et al. Exercise as a treatment for depression: a meta-analysis. J Affect Disord 2016;202:67-86.

5 Rice F, Eyre O, Riglin L, et al. Adolescent depression and the treatment gap. Lancet Psychiatry 2017;4:86-7.

6 Yang B, Wei J, Ju P, et al. Effects of regulating intestinal microbiota on anxiety symptoms: a systematic review. Gen Psychiatr 2019;32:e100056.

7 Borrow AP, Heck AL, Miller AM, et al. Chronic variable stress alters hypothalamic-pituitary-adrenal axis function in the female mouse. Physiol Behav 2019;209:112613.

8 Hadi S, Momenan M, Cheraghpour K, et al. Abdominal volume index: a predictive measure in relationship between depression/anxiety and obesity. Afr Health Sci 2020;20:257-65.

9 Thornton LM, Welch E, Munn-Chernoff MA, et al. Anorexia nervosa, major depression, and suicide attempts: shared genetic factors.. Suicide Life Threat Behav 2016;46:525-34.
10 Willner P, Towell A, Sampson D, et al. Reduction of sucrose preference by chronic unpredictable mild stress, and its restoration by a tricyclic antidepressant. Psychopharmacology 1987;93:358-64.

11 Yasuda K, Oh K, Ren B, et al. Biogeography of the intestinal mucosal and lumenal microbiome in the rhesus macaque. Cell Host Microbe 2015;17:385-91.

12 Mohd Shaufi MA, Sieo CC, Chong CW, et al. Deciphering chicken gut microbial dynamics based on high-throughput 16S rRNA metagenomics analyses. Gut Pathog 2015;7:4.

13 Zhang H, Ding L, Shen T, et al. HMGB1 involved in stress-induced depression and its neuroinflammatory priming role: a systematic review. Gen Psychiatr 2019;32:e100084.

14 Boku S, Nakagawa S, Toda H, et al. Neural basis of major depressive disorder: beyond monoamine hypothesis. Psychiatry Clin Neurosci 2018;72:3-12.

15 Bravo JA, Forsythe P, Chew MV, et al. Ingestion of Lactobacillus strain regulates emotional behavior and central GABA receptor expression in a mouse via the vagus nerve. Proc Natl Acad Sci U S A 2011;108:16050-5.

16 Herp S, Brugiroux S, Garzetti D, et al. Mucispirillum schaedleri antagonizes Salmonella virulence to protect mice against colitis. Cell Host Microbe 2019;25:681-94.

17 Barrett E, Ross RP, O'Toole PW, et al. $\gamma$-Aminobutyric acid production by culturable bacteria from the human intestine. $J$ Appl Microbiol 2012;113:411-7.

18 Humbel F, Rieder JH, Franc Y, et al. Association of alterations in intestinal microbiota with impaired psychological function in patients with inflammatory bowel diseases in remission. Clin Gastroenterol Hepatol 2020;18:2019-29. e11.

19 Lin X, Shao T, Wen X, et al. Combined effects of MSU crystals injection and high fat-diet feeding on the establishment of a gout model in C57BL/6 mice. Adv Rheumatol 2020;60:52.

20 Kang D-W, Adams JB, Gregory AC, et al. Microbiota transfer therapy alters gut ecosystem and improves gastrointestinal and autism symptoms: an open-label study. Microbiome 2017;5:10.

21 Tomova A, Husarova V, Lakatosova S, et al. Gastrointestinal microbiota in children with autism in Slovakia. Physiol Behav 2015;138:179-87.

22 Finegold SM, Downes J, Summanen PH. Microbiology of regressive autism. Anaerobe 2012;18:260-2.

23 Liu RT, Rowan-Nash AD, Sheehan AE, et al. Reductions in antiinflammatory gut bacteria are associated with depression in a sample of young adults. Brain Behav Immun 2020;88:308-24.

24 Getachew B, Tizabi Y. Effects of C-terminal domain of the heavy chain of tetanus toxin on gut microbiota in a rat model of depression. Clin Pharmacol Transl Med 2019;3:152-9.

25 Xie R, Jiang P, Lin L, et al. Oral treatment with Lactobacillus reuteri attenuates depressive-like behaviors and serotonin metabolism alterations induced by chronic social defeat stress. J Psychiatr Res 2020;122:70-8.

$26 \mathrm{Kim}$ Y-H, Nagata R, Ohkubo A, et al. Changes in ruminal and reticular $\mathrm{pH}$ and bacterial communities in Holstein cattle fed a high-grain diet. BMC Vet Res 2018;14:310.

27 Patrone V, Minuti A, Lizier M, et al. Differential effects of coconut versus soy oil on gut microbiota composition and predicted metabolic function in adult mice. BMC Genomics 2018;19:808.

28 Ling Z, Li Z, Liu X, et al. Altered fecal microbiota composition associated with food allergy in infants. Appl Environ Microbiol 2014:80:2546-54.

29 Song J, Zhou N, Ma W, et al. Modulation of gut microbiota by chlorogenic acid pretreatment on rats with adrenocorticotropic hormone induced depression-like behavior. Food Funct 2019;10:2947-57.

$30 \mathrm{Li} \mathrm{Y,} \mathrm{Lu} \mathrm{X,} \mathrm{Li} \mathrm{X,} \mathrm{et} \mathrm{al.} \mathrm{Effects} \mathrm{of} \mathrm{Agaricus} \mathrm{blazei} \mathrm{Murrill}$ polysaccharides on hyperlipidemic rats by regulation of intestinal microflora. Food Sci Nutr 2020;8:2758-72.

31 Brummelte S, Pawluski JL, Galea LAM. High post-partum levels of corticosterone given to dams influence postnatal hippocampal cell proliferation and behavior of offspring: a model of post-partum stress and possible depression. Horm Behav 2006;50:370-82.

32 McEwen BS. Stress and hippocampal plasticity. Annu Rev Neurosci 1999:22:105-22.

33 Middeldorp J, Hol EM. GFAP in health and disease. Prog Neurobiol 2011;93:421-43.

34 Namiki J, Suzuki S, Masuda T, et al. Nestin protein is phosphorylated in adult neural stem/progenitor cells and not endothelial progenitor cells. Stem Cells Int 2012;2012:430138.

35 Barkho BZ, Song H, Aimone JB, et al. Identification of astrocyteexpressed factors that modulate neural stem/progenitor cell differentiation. Stem Cells Dev 2006;15:407-21. 
36 Mächler P, Wyss MT, Elsayed M, et al. In vivo evidence for a lactate gradient from astrocytes to neurons. Cell Metab 2016;23:94-102.

37 Woelfer M, Kasties V, Kahlfuss S, et al. The role of depressive subtypes within the neuroinflammation hypothesis of major depressive disorder. Neuroscience 2019;403:93-110.

$38 \mathrm{Yu}$ T, Zhang $\mathrm{X}$, Shi $\mathrm{H}$, et al. P2Y12 regulates microglia activation and excitatory synaptic transmission in spinal lamina II neurons during neuropathic pain in rodents. Cell Death Dis 2019;10:165.
39 Pellegrini C, Antonioli L, Calderone V, et al. Microbiota-gutbrain axis in health and disease: is NLRP3 inflammasome at the crossroads of microbiota-gut-brain communications? Prog Neurobiol 2020;191:101806.

40 Camargo A, Dalmagro AP, Platt N, et al. Cholecalciferol abolishes depressive-like behavior and hippocampal glucocorticoid receptor impairment induced by chronic corticosterone administration in mice. Pharmacol Biochem Behav 2020;196:172971.

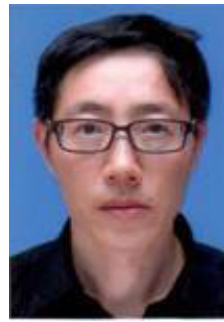

Dr Zejian Wang obtained a PHD degree from Shanghai Jiao Tong University, China in 2003. He is currently an associate professor at Shanghai Jiao Tong University, Shanghai, China. He has participated in and presided over two National Natural Science Foundation of China. He is also a member of Shanghai Pharmacological society in China. He has attended and presented in various national and international conferences. His main research interests include pathogenesis of depression, Alzheimer's Disease and cerebral neural activity. 